\title{
Membrane-bound catechol-O-methyl transferase in cortical neurons and glial cells is intracellularly oriented
}

\section{Björn H. Schott1,2,3* , Renato Frischknecht', Grazyna Debska-Vielhaber ${ }^{1,2}$, Nora John ${ }^{1}$, Gusalija Behnisch', Emrah Düzel ${ }^{2,4,5}$, Eckart D. Gundelfinger ${ }^{1}$ and Constanze I. Seidenbecher ${ }^{1}$}

\author{
' Department of Behavioral Neurology, Leibniz Institute for Neurobiology, Magdeburg, Germany \\ 2 Department of Neurology, Otto von Guericke University, Magdeburg, Germany \\ ${ }^{3}$ Department of Psychiatry, Campus Mitte, Charité University Hospital, Berlin, Germany \\ ${ }^{4}$ Department of Cognitive Neurology and Dementia Research, Otto von Guericke University, Magdeburg, Germany \\ ${ }_{5}^{5}$ Institute for Cognitive Neuroscience, University College London, London, UK
}

Edited by:

Ming D. Li, University of Virginia, USA

Reviewed by:

Ming D. Li, University of Virginia, USA

Junran Cao, University of Virginia, USA

Ludger Schoels, University of Tübingen,

Germany

\section{*Correspondence:}

Björn H. Schott, Department of

Behavioral Neurology, Leibniz Institute for Neurobiology, Brenneckestr. 6,

39118 Magdeburg, Germany.

e-mail: bschott@neuro2.med.

uni-magdeburg.de
Catechol-O-methyl transferase (COMT) is involved in the inactivation of dopamine in brain regions in which the dopamine transporter (DAT1) is sparsely expressed. The membrane-bound isoform of COMT (MB-COMT) is the predominantly expressed form in the mammalian central nervous system (CNS). It has been a matter of debate whether in neural cells of the CNS the enzymatic domain of MB-COMT is oriented toward the cytoplasmic or the extracellular compartment. Here we used live immunocytochemistry on cultured neocortical neurons and glial cells to investigate the expression and membrane orientation of native COMT and of transfected MB-COMT fused to green fluorescent protein (GFP). After live staining, COMT immunoreactivity was reliably detected in both neurons and glial cells after permeabilization, but not on unpermeabilized cells. Similarly, autofluorescence of COMT-GFP fusion protein and antibody fluorescence showed overlap only in permeabilized neurons. Our data provide converging evidence for an intracellular membrane orientation of MB-COMT in neurons and glial cells, suggesting the presence of a DAT1-independent postsynaptic uptake mechanism for dopamine, prior to its degradation via COMT.

Keywords: catechol- $O$-methyl transferase, membrane, neuronal cell culture, immunocytochemistry, dopamine

\section{INTRODUCTION}

Catechol-O-methyl transferase (COMT, EC_number 2.1.1.6) is involved in the degradation of dopamine and other catecholamines by transferring a methyl group donated by $S$-adenosyl-methionine onto a phenolic hydroxyl group. COMT is expressed in two isoforms, a soluble form (S-COMT), which is highly expressed in peripheral tissues, such as the liver, and a membrane-bound form (MB-COMT).MB-COMT is the predominantly expressed COMT isoform in the mammalian central nervous system (CNS; Rivett et al., 1983a, b; Reenilä and Männistö, 2001; Chen et al., 2004).

Converging evidence from animal studies and human post mortem investigations raised the hypothesis that COMT is particularly important for dopamine inactivation when dopamine uptake by the presynaptic dopamine transporter (DAT1) is inhibited or in brain regions in which DAT1 is sparsely expressed, such as the prefrontal cortex (PFC; Karoum et al., 1994; Sesack et al., 1998; Matsumoto et al., 2003). The pivotal role of COMT in the control of prefrontal dopamine levels has attracted considerable attention in neuropsychiatric research,

Abbreviations: COMT; catechol-O-methyl transferase; DAT1, dopamine transporter [1]; DIV, days in vitro; DMEM, Dulbecco's Modified Eagle Medium; ECL, enhanced chemiluminescence system; EMT, extraneuronal monoamine transporter; GFAP, glial fibrillary acidic protein; GFP, green fluorescent protein; HBSS, Hank's balanced salt solution; MAP2, microtubule-associated protein 2; MB-COMT, membrane-bound COMT; NBM, Neurobasal medium; NET, norepinephrine transporter; NgCAM, neuron-glia cell adhesion molecule; NrCAM, neuronal cell adhesion molecule; PFA, paraformaldehyde; PFC, prefrontal cortex; PMAT, plasma membrane amine transporter; RT, room temperature; S-COMT, soluble COMT; TBS, Tris-buffered saline. and genetic variations of COMT have been implicated in an increased individual risk for schizophrenia (Egan et al., 2001; for a review see Williams et al.,2007) and in PFC-dependent cognitive function in both healthy humans and patients with schizophrenia (Egan et al., 2001; Schott et al., 2006; Tan et al., 2007; Diaz-Asper et al., 2008).

DAT1-independent function of COMT would require either extracellular activity of the enzyme or a DAT1-independent catecholamine uptake, which might be postsynaptic or glial (Tunbridge et al., 2006a). Previous studies have demonstrated that COMT is highly expressed in glial cells and also perisynaptically in neurons (Rivett et al., 1983b; Karhunen et al., 1995a; Matsumoto et al., 2003). However, these studies do not provide information on the membrane orientation of the enzyme. As deduced from the primary structure, MB-COMT has a single hydrophobic region, which might act as an internal start-transfer sequence for non-cleaved signal anchor proteins. Different computer-assisted membrane topology prediction routines based on the classical von Heijne algorithms (Elofsson and von Heijne, 2007) lead to non-uniform predictions: either a type I transmembrane topology with the $\mathrm{N}$-terminus extra- and the C-terminus intracellularly or a type II configuration with the opposite orientation. In non-neuronal cells heterologously expressing MB-COMT, COMT immunoreactivity was only detectable after permeabilization of cells (Ulmanen et al., 1997). This study also suggested that COMT overexpressed in neurons using a viral expression system was primarily located at intracellular organelle membranes rather than at the plasma membrane. An electron microscopic study, however, did show that 
COMT immunoreactivity could be observed at the dendritic plasma membrane of parietal cortex neurons and was present at synaptic membranes (Karhunen et al., 1995b), but the orientation of the immunoreactive domain could not be resolved by that study.

On the other hand, microdialysis studies have provided evidence for high concentrations of COMT-dependent dopamine metabolites in brain regions with low DAT1 expression or under conditions of pharmacological DAT1 inhibition (Karoum et al., 1994; Huotari et al., 1999). Furthermore, the different temporal patterns of tonic and phasic dopamine action in the striatum and the PFC have been suggested to result, in part, from extracellular activity of COMT (Bilder et al., 2004). To provide better understanding of the relationship between dopamine action in the striatum and the PFC, it thus seems critical to elucidate the membrane orientation of COMT in the CNS (Tunbridge et al., 2006a).

Here, we used live staining of rat primary neural cultures to investigate the orientation of the MB-COMT catalytic domain in neurons and glial cells. Both, immunostaining of natively expressed COMT in cortical cultures, and co-localization studies of COMTGFP fusion constructs were performed.

\section{MATERIALS AND METHODS ANTIBODIES}

The rabbit anti-COMT polyclonal antibody (Chemicon, AB5873) was used at a concentration of 1:500. The rabbit polyclonal anti-GFP antibody (Abcam, ab6556) was used at concentrations of 1:5,000 and 1:1,000 for Western blotting and immunocytochemistry, respectively. Mouse monoclonal antibodies against microtubule-associated protein 2 (MAP2; 1:1,000) and glial fibrillary acidic protein (GFAP; 1:500) were also obtained from Abcam (ab28032, ab10062). The anti-Thy1.1 mouse monoclonal antibody (Millipore, MAB1406) was used at a concentration of 1:400. Brevican antiserum from guinea pig was used as described previously (John et al., 2006).

For Western blotting, horseradish peroxidase (HRP)-coupled secondary antibodies from the enhanced chemiluminescence system (ECL; Amersham Biosciences) and the murine anti-rabbit IgG peroxidase-coupled antibody (Sigma, A-1949) were employed according to manufacturers' protocols. For fluorescence microscopy, secondary antibodies conjugated to Alexa-488, Alexa-568, and Alexa-647 (Molecular Probes, A11073, A11035, A21236, A11031) were used at a concentration of 1:1,000.

\section{GENERATION OF COMT-GFP FUSION CONSTRUCTS, TRANSFECTION, AND CELL FRACTIONATION}

Because the hydrophobic peptide that putatively functions as a transmembrane domain in MB-COMT is located toward the $\mathrm{N}$-terminus of the protein, green fluorescent protein (GFP) moieties were fused to the C-terminus (Figure 4A). The cDNA of human MB-COMT and S-COMT was amplified from a fetal human CDNA library (Stratagene) using nested PCR and cloned into the EcoRI and BamHI restriction sites of the pEGFP-N1 eukaryotic expression vector (Clontech). The Met codon at amino acid position 51 of human MB-COMT cDNA (NM_000754), which also serves as the start codon for S-COMT, was mutated to GTG (Val) or GCG (Ala), using PCR-based site-directed mutagenesis. Primers used for cloning and mutagenesis are displayed in Table 1. Mutated constructs were also cloned into pEGFP-N1, using the same restriction sites. The base sequences of the inserts and the flanking regions of the vector were verified by custom sequencing into both directions (SeqLab).

To verify the correct expression of the COMT-GFP fusion constructs, COS7 cells were transfected with the constructs, using the PolyFect transfection reagent (Qiagen, Chatsworth, CA, USA) according to manufacturer's protocols. After incubation at $37^{\circ} \mathrm{C}$ for $36 \mathrm{~h}$, cells were visually inspected for green fluorescence, and harvested in Tris-buffered saline (TBS). Protein fractionation and Western blotting were conducted as described previously (Seidenbecher et al., 2002), with minor modifications. Briefly, cells were homogenized in TBS containing a protease inhibitor cocktail (Complete; Boehringer Mannheim, Germany) and centrifuged at $12,000 \mathrm{~g}$ for $15 \mathrm{~min}\left(4^{\circ} \mathrm{C}\right)$. The supernatant was kept as cytosolic fraction ( $\mathrm{S} 1$; including microsomes), and the pellet was rehomogenized in TBS containing 1\% Triton X-100 and centrifuged at $20,000 \mathrm{~g}$ for $15 \mathrm{~min}\left(4^{\circ} \mathrm{C}\right)$, yielding the S2 fraction containing detergent-soluble membrane proteins and the pellet $(\mathrm{P})$ containing Triton-insoluble membrane or cytoskeletal proteins. All obtained protein fractions were solubilized with sodium dodecyl sulfate (SDS) and mercaptoethanol. The whole-cell homogenate and the fractionated proteins were separated by SDS-PAGE on 5-20\% gels under fully reducing conditions and transferred onto nitrocellulose according to standard protocols. Western blots were incubated overnight with the primary antibody, and processed using the ECL system according to the manufacturer's protocol.

Table 1 | Primers for cloning and mutagenesis.

\begin{tabular}{|c|c|c|}
\hline & Forward-primer, reverse-primer & $T_{\mathrm{A}}$ \\
\hline \multirow[t]{2}{*}{ Cloning (first PCR) } & cCOMT-out-f: CCTGCTCAGAGGTGCTTTGAAG & 52 \\
\hline & cCOMT-out-r. TCAGTACCAGGCTGGGTGAGA & \\
\hline \multirow{2}{*}{ Cloning (second PCR) } & cS-COMT-f: AAGAATCCTCATGGGTGACACCAAGGAGCAG & \\
\hline & cCOMT-r. ATGGATCCGCGGGCCCTGCTTCGCTGCCTGG & \\
\hline \multirow[t]{2}{*}{ Mutagenesis M51V } & cCOMT51 Val-f. CAACCTGCTCGTGGGTGACACCAAGG & 58 \\
\hline & cCOMT51 Val-r. CCTTGGTGTCACCCACGAGCAGGTTG & \\
\hline
\end{tabular}

$T_{A}$ : annealing temperature $\left({ }^{\circ} \mathrm{C}\right)$. 


\section{PRIMARY NEURONAL CULTURES}

Animal handling and preparation of neuronal cell cultures were carried out in accordance with the guidelines of the Committee for Animal Protection of the Leibniz Institute for Neurobiology, Magdeburg. Preparation of cortical neurons was performed as described previously (Banker and Goslin, 1988), with minor modifications. Brains from embryonic rats (E16 for transfections, E18 for native immunocytochemistry) were prepared under Hank's balanced salt solution (HBSS; $+\mathrm{Ca}^{2+}, \mathrm{Mg}^{2+}$ ), and the cortex (excluding the olfactory bulb) was dissected, washed in HBSS $\left(-\mathrm{Ca}^{2+}, \mathrm{Mg}^{2+}\right)$ and incubated in HBSS containing trypsin for $5 \mathrm{~min}$ $\left(37^{\circ} \mathrm{C}\right)$. After trypsin removal cells were carefully dispersed under DMEM cell culture medium (10\% fetal calf serum [FCS], $1 \mathrm{mM}$ Na-Pyruvate, $1 \mathrm{mM} \mathrm{Gln}$ ), and the cell suspension was diluted to a density of 1,500 cells/ $\mu$ land seeded onto poly-D-lysine-coated $18 \mathrm{~mm}$ cover slips. After $24 \mathrm{~h}, \mathrm{DMEM}$ was replaced by neurobasal medium $(\mathrm{NBM})$, containing $1 \times \mathrm{B} 27,1 \mathrm{mM}$ sodium pyruvate, $1 \times$ Albumax and penicillin and streptomycin as antibiotics. Medium for transfected cells additionally contained cytosine arabinoside (AraC, final concentration $15 \mu \mathrm{M}$ ), to limit excessive glial cell proliferation.

For Western blot analysis of COMT expression in neurons and glial cells, 250,000 rat cortical neurons prepared from E16-18 rats were plated in a $3-\mathrm{cm}$ culture dish and cultured for 21 days. Glial cells were prepared from brains of rat pups on postnatal day 2 and grown in $3 \mathrm{~cm}$ culture dishes until confluence. Cell lysates were prepared by incubation of the cultures in $500 \mathrm{ml} \mathrm{HBSS}$ containing $1 \%$ Triton $\mathrm{X}-100$ and $1 \%$ SDS at $4^{\circ} \mathrm{C}$ for $15 \mathrm{~min}$.

Transfection of neurons with expression vectors containing COMT-GFP fusion constructs was performed on days 7-10 in vitro (DIV 7-10) using the Effectene transfection reagent (Qiagen, Chatsworth, CA) according to the manufacturer's protocol. No later than $12 \mathrm{~h}$ after transfection, the medium was replaced with fresh NBM. An additional population of cells was transfected with a previously described construct of GFP integrated into the extracellular portion of the NgCAM-related cell adhesion molecule NrCAM (NrCAM-GFP, Thoumine et al., 2005), using the same transfection protocol. The NrCAM-GFP construct served as a positive control for live staining of unpermeabilized cells.

\section{IMMUNOCYTOCHEMICAL PROCEDURES}

COS7 cells were grown on poly-D-lysine-coated coverslips, and membrane orientation was tested using immunocytochemistry, as described below, with minor modifications (i.e., permeabilization of COS7 cells was performed using methanol/acetone instead of digitonin).

\section{Immunocytochemistry of endogenous COMT}

For live staining, cells at DIV 16-23 were incubated with the primary antibodies at $37^{\circ} \mathrm{C}$ for $15 \mathrm{~min}$. Digitonin (final concentration: $5 \mu \mathrm{g} / \mathrm{ml}$ for neurons, $30 \mu \mathrm{g} / \mathrm{ml}$ for glial cells) was used for permeabilization of cell membranes. Cells were then fixed (4\% paraformaldehyde [PFA] for $10 \mathrm{~min}$ at room temperature [RT]), washed three times in $1 \times$ PBS and blocked with $10 \%$ horse serum $+25 \mathrm{mM}$ glycine for $60 \mathrm{~min}$. After blocking, cells were briefly rinsed $(1 \times$ PBS) and incubated with the secondary antibodies in $5 \%$ horse serum at RT for $60 \mathrm{~min}$. Following a further washing step
( $3 \times$ in $1 \times$ PBS), coverslips were mounted in Mowiol (Hoechst). For staining of fixed cells, incubation with the primary antibody was performed after the blocking/permeabilization step at RT for 30-60 min, with digitonin or $0.1 \%$ Triton X-100. All other steps were performed as described above. An antibody directed against the extracellular matrix proteoglycan Brevican was used as positive control to verify the live staining procedure of untransfected cells (Frischknecht et al., 2009).

\section{Immunocytochemistry of COMT-GFP-transfected cells}

We performed immunostainings for GFP on the transfected cells as live stainings, with or without permeabilization of membranes using digitonin, as described above. Additional stainings of permeabilized cells were performed after blocking/permeabilization with PFA (see above for details).

For live staining at DIV 8-12, 24-48 h after transfection, cells seeded on cover slips were incubated with the primary antibodies, diluted in $\mathrm{NBM}$, under $\mathrm{CO}_{2}$ at $37^{\circ} \mathrm{C}$ for $15 \mathrm{~min}$. To permeabilize cell membranes, digitonin solution was added to a final concentration of $10-15 \mu \mathrm{g} / \mathrm{ml}$. After live staining, cells were fixed with ice-cold $4 \%$ PFA and $4 \%$ sucrose.

Cells were examined under a Zeiss Axioplan 2 microscope equipped with a SpotRT cooled CCD camera or a Leica DM-RXE fluorescence microscope, using the Spot software. Surface expression of COMT and NrCAM was quantified using ImageJ (National Institutes of Health, Bethesda, MD, USA). For the profile plot, fluorescence intensities were measured along a line with diameter of $500 \mathrm{~nm}$ using ImageJ.

\section{STATISTICAL ANALYSIS}

To compare fluorescence intensity of live-stained, unpermeabilized and fixed, permeabilized cells statistically, we computed the average fluorescence intensities of independent cells $(N=8$ per group for endogenous COMT; $N=6$ per group for cells overexpressed COMT-GFP fusion constructs; $N=5$ per group for overexpressed COMT-NrCAM fusion constructs).

Statistical analysis was carried out using the algorithms implemented in VassarStats (http://faculty.vassar.edu/lowry/VassarStats. html). To quantify fluorescence of cells stained against native COMT, the raw mean gray values (arbitrary units) of live-stained cells and fixed cells were compared to those of cells stained with secondary antibody only, using a one-way ANOVA with the factor treatment (fixed, live, no primary). Antibody fluorescence of overexpressed COMT and NrCAM was normalized to the GFP autofluorescence of the fusion constructs, and the relative antibody fluorescence was compared using a two-way ANOVA with the factors construct (COMT-GFP, NrCAM-GFP) and treatment (live, fixed). ANOVAs were followed by post hoc T-test to verify directionality of the effects.

\section{RESULTS}

\section{EXPRESSION OF NATIVE COMT IN NEURONS AND GLIAL CELLS}

Co-staining with antibodies against COMT and MAP2 or GFAP showed that COMT immunoreactivity was reliably detectable in both, cultured cortical neurons (Figures 1C,D,G,H) and glial cells (Figures 1K,L,O,P). In fixed and permeabilized MAP2-positive neurons, the COMT signal appears concentrated in the soma at perinuclear 


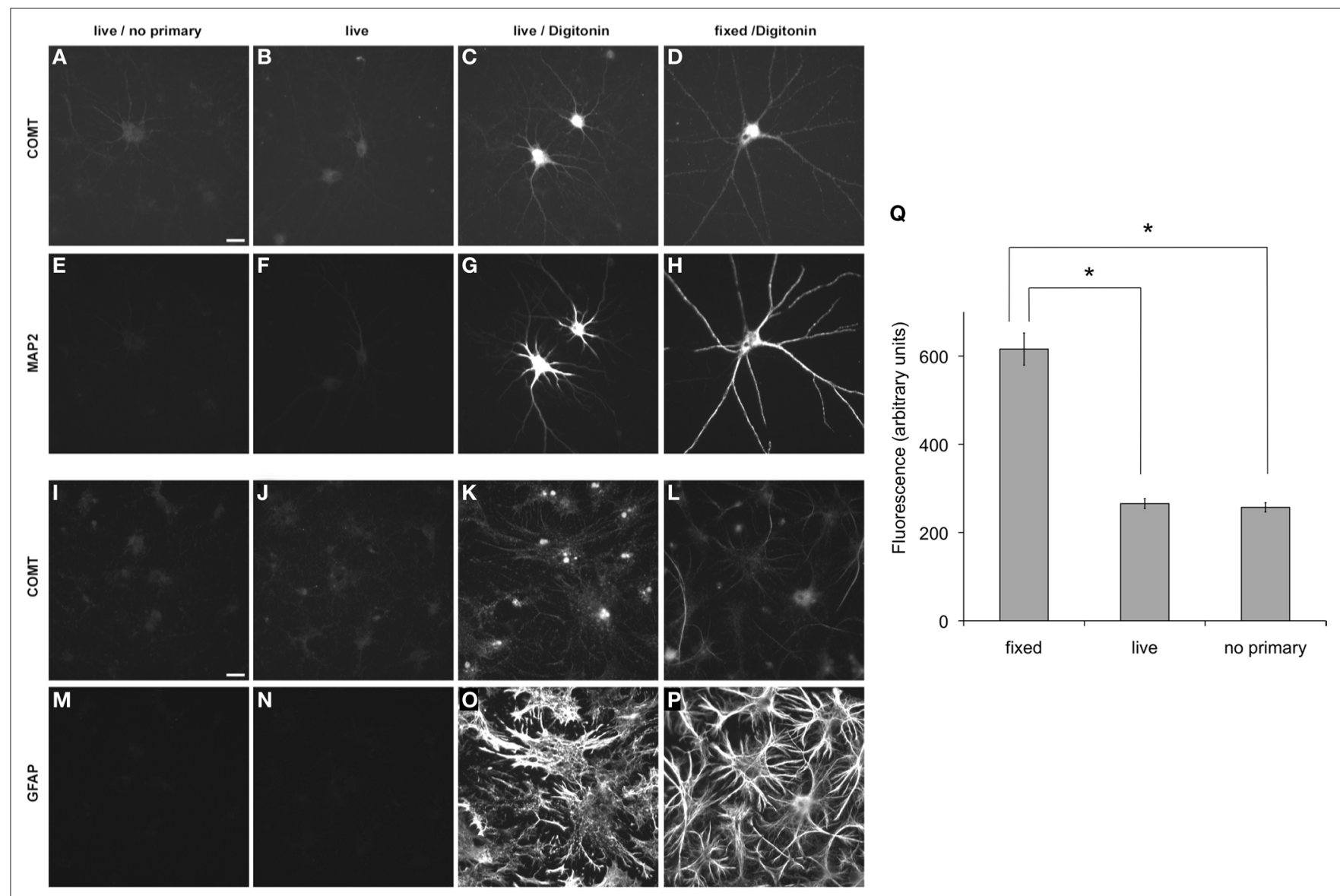

FIGURE 1 | Detection of endogenous COMT in primary cortical neurons and glial cells at DIV 23 under permeabilized or non-permeabilized conditions. (A-D,I-L) Staining for COMT. (E-H) Co-stainings with an antibody against the neuronal marker MAP2. (M-P) Co-stainings with an antibody against the glial marker GFAP. (A,E,I,M) Controls without primary antibody. (B,F,J,N) Neurons and glial cells stained live for COMT immunoreactivity. $\mathbf{( C , G , K , O )}$
Neurons and glial cells stained live for COMT immunoreactivity after permeabilization. (D,H,L,P) Neurons and glial cells stained for COMT immunoreactivity after fixation and permeabilization. Size bars denote $20 \mu \mathrm{m}$. (Q) Statistical comparison of fluorescence intensity showed a significant difference between fixed, permeabilized cells and live-stained, unpermeabilized cells, while the latter did not differ from background fluorescence (no primary). sites, but is also present in dendrites (Figure 1D). Similarly, after fixation, GFAP-positive astroglia exhibited reliable COMT immunoreactivity with a filamentous appearance (Figure 1L).

To obtain first information about intra- vs. extracellular localization of the COMT epitopes, live staining of permeabilized and unpermeabilized cells was performed. Immunocytochemistry on living cells did not yield a staining above background neither in neurons nor in glial cells (Figures 1A,B,I,J). Only after membrane permeabilization, specific COMT immunoreactivity was detected suggesting an intracellular localization of the epitopes (compare Figure 1B vs. Figures 1C,D,J vs. Figures 1K,L). The feasibility of the live staining procedure on unpermeabilized cells was confirmed by live staining of cultured cells with an antibody against the extracellular matrix proteoglycan Brevican, which revealed the typical perineuronal distribution of Brevican immunoreactivity described previously (data not shown, see John et al., 2006; Frischknecht et al., 2009). The detectability of marker proteins GFAP or MAP2 proves the unpermeabilized (Figures 1F,N) or permeabilized (Figures $1 \mathrm{G}, \mathrm{H}, \mathrm{O}, \mathrm{P})$ state of the cells, with unpermeabilized cells showing only background fluorescence (Figures 1E,M).
We next quantified fluorescence intensities of live-stained unpermeabilized and fixed permeabilized neurons as compared to neurons stained with secondary, but no primary antibody ( $N=8$ cells per group). A one-way ANOVA revealed a significant between-group difference $\left(F_{2,21}=80.41, p<0.0001\right)$, and post hoc $T$-tests confirmed that fluorescence of live-stained cells did not differ from the background fluorescence of cells treated without primary antibody, while both exhibited significantly lower fluorescence than permeabilized cells (live $>$ no primary: $T_{14}=0.56$; $p=0.292$; fixed $>$ live: $T_{14}=9.19, p<0.0001$; fixed $>$ no primary: $T_{14}=9.46, p<0.0001$; see Figure 1Q).

To obtain a more detailed view of the cellular distribution of COMT, cortical neurons were first subjected to live staining against Thy-1, a GPI-anchored surface protein from the Ig-superfamily, and then fixed, permeabilized and stained against COMT (Figures 2A-D). A profile plot through a dendritic branch showed two peaks in Thy-1 fluorescence, representing Thy-1-labeling at the extracellular side of the plasma membrane (Figures 2D,E). Similarly, two lateral peaks of increased COMT fluorescence were observed near the plasma membrane. These were, however, slightly 

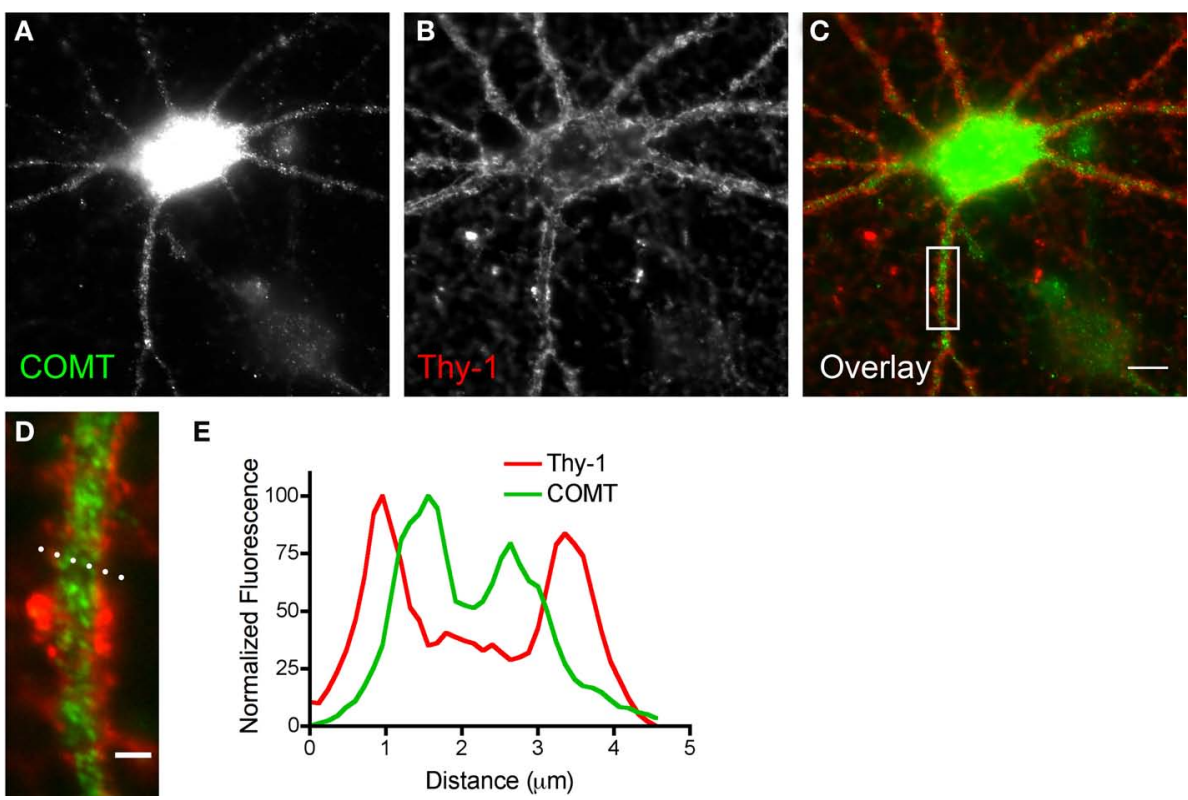

$\mathbf{E}$

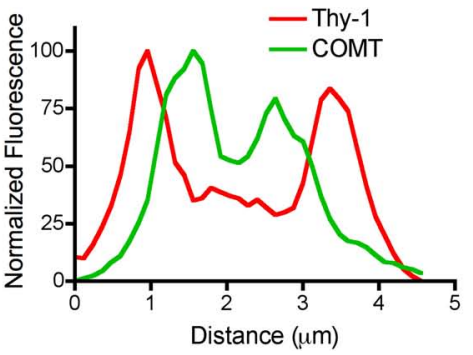

FIGURE 2 | Catechol-O-methyl transferase staining is enriched in the vicinity of the neuronal membrane. Dissociated cortical neurons at DIV21 stained with an anti-COMT antibody after permeabilization (A). Strongest immunofluorescence was found in the cell body. Staining in the dendrites had diffuse and dot-like appearance. Live staining against Thy- 1 was used to label the neuronal surface (B), which did hardly overlap with COMT as seen in the overlay (C) or the high magnification image of the dendritic segment boxed in (C). (D) In order to analyze COMT distribution in more detail we performed a profile plot through a dendritic segment. The dotted line in (D) represents the position of the profile plot shown in (E). For Thy-1 (red line), two peaks in fluorescence were found representing the outer boundary of the neuronal membrane. Similarly, two peaks were found for COMT fluorescence (green line), also pointing toward a plasma membrane association of COMT. However, the fluorescence is slightly shifted toward the cytoplasm indicating, as expected, an intracellular localization of COMT epitopes (scale bars: $10 \mu \mathrm{m}$ in $\mathbf{C}$ $2 \mu \mathrm{m}$ in D) shifted toward the intracellular compartment, suggesting localization of COMT epitopes at the intracellular side of the plasma membrane (Figures 2D,E). Furthermore, diffuse staining was found within the dendrite, most likely due to the presence of S-COMT and MB-COMT at intracellular membranes (Karhunen et al., 1995a,b; Ulmanen et al., 1997).

Because the antibody recognizes both, S-COMT and MB-COMT signals, the immunostainings do not provide information about potentially differential expression of the isoforms in neurons or glial cells. We therefore employed Western blot analyses to investigate potentially dissociable expression patterns of COMT isoforms in neurons and glia. Western blotting revealed predominant expression of MB-COMT in neurons and similar amounts of S-COMT and MB-COMT in glial cells (Figure 3). Furthermore, a larger COMT-immunoreactive band was observed in both cell types and in brain homogenate at approximately $39 \mathrm{kDa}$, probably representing a novel COMT isoform (Tunbridge et al., 2006b). In the brain extract, several smaller immunoreactive bands were detected with the antibody, possibly reflecting partially degraded COMT protein.

\section{MEMBRANOUS LOCALIZATION OF MUTATED MB-COMT-GFP FUSION CONSTRUCTS}

To circumvent the obstacle that the polyclonal antibodies against COMT might potentially be not suitable for live detection of the native antigen, we performed co-localization studies of COMTGFP autofluorescence and antibody fluorescence of immunostaining against the GFP epitope fused to the C-terminal end of
MB-COMT (Figure 4A). To avoid co-expression of S-COMT-GFP, the Met codon at position 51 was mutated to Ala or Val (Ulmanen et al., 1997).

All COMT-GFP fusion constructs could be reliably expressed in COS7 cells (Figure 4B) as indexed by GFP autofluorescence. Western blotting of homogenized COS7 cells revealed that the mutagenesis at amino acid position 51 led to an exclusive expression of MB-COMTGFP in absence of S-COMT-GFP (Figure 4C), and the protein fractionation analysis further showed that both MB-COMT-Ala and MB-COMT-Val were mostly localized to membranous compartments of COS7 cells (Figure 4D) indicating correct intracellular processing of the mutated chimeric proteins.

\section{MEMBRANE ORIENTATION OF COMT-GFP FUSION CONSTRUCTS}

The cellular localization of the COMT-GFP fusion protein was first investigated in COS7 cells. Cells permeabilized with methanol/ acetone showed basically an exact overlap of antibody fluorescence with the autofluorescence of the GFP fusion constructs. After live staining of unpermeabilized cells, no specific antibody fluorescence was detectable, and there was consequently no relevant overlap of antibody fluorescence and GFP autofluorescence (data not shown). This finding is in agreement with previously reported intracellular orientation of MB-COMT in non-neuronal cells (Ulmanen et al., 1997).

As functionality of cellular sorting signals and membrane topology might be cell-type specific and different in cells endogenously or heterologously expressing COMT, we investigated the possibility of a potential neuron-specific extracellular orientation of MB-COMT. To 


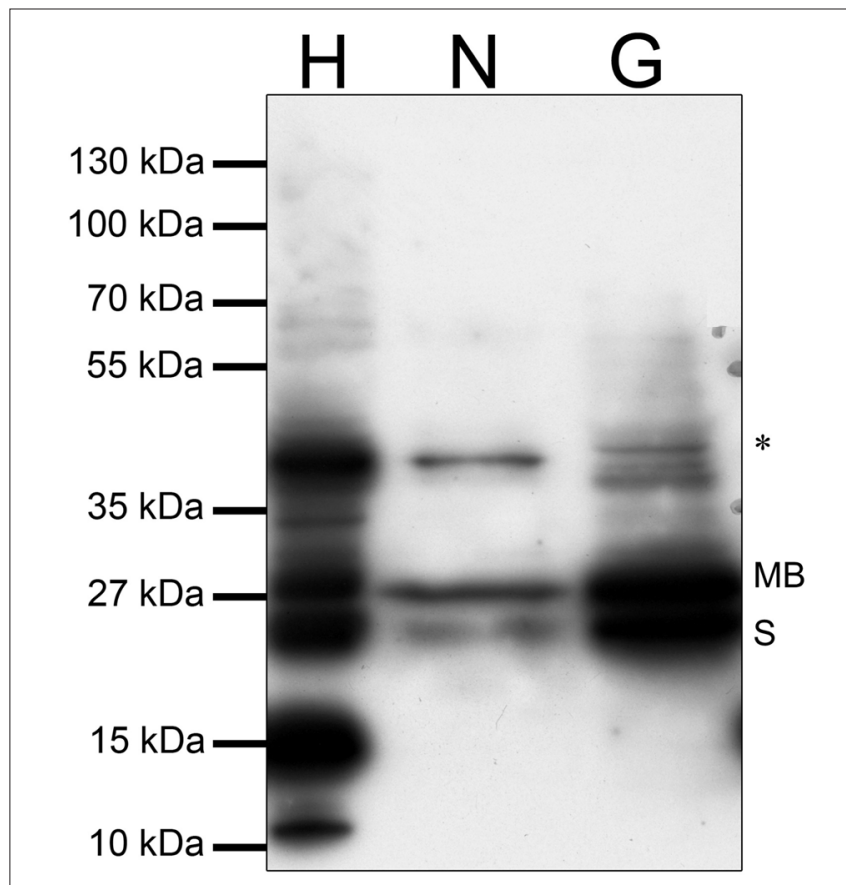

FIGURE 3 | Discrimination of COMT isoforms in Western blotting. In the mouse forebrain homogenate $(\mathrm{H})$ and in cultured glial cells $(\mathrm{G}), \mathrm{MB}-\mathrm{COMT}$ and S-COMT were expressed at similar levels, while MB-COMT was the predominant isoform in cultured cortical neurons at DIV 21 (N). MB, MB-COMT; S, S-COMT; the asterisk $\left(^{*}\right)$ denotes a b and most likely corresponding to a previously described novel COMT isoform (Tunbridge et al., 2006b). Note that the exposition time of the blot was adjusted to visualize the faint bands in the neuronal culture $(\mathrm{N})$, which leads to overexposition of strong bands in the homogenate and the glial culture lanes $(H, G)$.

this end, embryonal cortex neurons from rats (E16) were transfected with the COMT-GFP construct at DIV 7-10. We immunostained transfected neurons either live, with or without permeabilization by digitonin, or fixed and permeabilized with Triton X-100. Neurons transfected with a GFP fusion construct of the cell-surface adhesion molecule NrCAM were used as positive controls for live staining of an extracellular GFP epitope. As expected, the control neurons transfected with NrCAM-GFP showed reliable overlap of GFP autofluorescence and antibody fluorescence with and without permeabilization. In contrast, overlap of COMT-GFP autofluorescence and antibody fluorescence was detectable only after permeabilization of the cells, providing further evidence for intracellular orientation of MB-COMT in neurons (Figure 5A). For statistical comparison, the antibody fluorescence intensity of fixed permeabilized and livestained unpermeabilized cells was normalized to the GFP autofluorescence. We performed a two-way ANOVA with construct (COMT $[N=2 \times 6]$ vs. $\mathrm{NrCAM}[N=2 \times 5]$ ) and treatment (fixed permeabilized vs. live unpermeabilized) as factors. There was a significant main effect of treatment $\left(F_{1,1,18}=54.76, p<0.0001\right)$, reflecting overall stronger antibody fluorescence for fixed cells, and a significant construct by treatment interaction $\left(F_{1,1,18}=12.27, p=0.0025\right)$, reflecting a significant difference in antibody fluorescence for COMT vs. NrCAM in unpermeabilized, but not in fixed cells. This difference was confirmed by a post hoc $T$-test (COMT < NrCAM: T9 $=-5.34$, $p=0.0002$; see Figure 5B).

\section{DISCUSSION}

The intracellular localization and orientation of MB-COMT in neurons and glial cells is critical for the understanding of the precise mechanism by which COMT influences dopamine availability in brain structures with low dopamine transporter activity.

Building on previous studies showing the presence of COMT at postsynaptic membranes (Karhunen et al., 1995b) and the intracellular localization of COMT epitopes in non-neuronal cells (Ulmanen et al., 1997), our present immunocytochemical localization studies of native neuronal and glial COMT and of GFP-fused MB-COMT in primary neural cell cultures provide converging evidence for an intracellular orientation of neural MB-COMT. Profile plot revealed that COMT immunoreactivity did peak at dendritic plasma membranes, but was shifted intracellularly relative to the - known extracellular - fluorescence of the Thy-1 epitope. In our overexpression experiments, the GFP moiety was fused to the C-terminus of MB-COMT, distal to the putative transmembrane domain and had thus the same membrane orientation as the catalytic domain. One potential caveat might be that the inverse approach, fusing GFP to the N-terminus of MB-COMT was attempted, but the resulting construct formed aggregates and exerted cytotoxic effects. It should be noted, though, that such an outcome was not unexpected, as fusing the relatively large GFP to the small $\mathrm{N}$-terminus (six amino acids) was quite likely to profoundly affect posttranslational intracellular processing of the protein in an unpredictable fashion. On the other hand, such effects would be much less expected when fusing the GFP tag to the C-terminus. Extracellular action of neuronal or glial MB-COMT is thus unlikely to contribute to metabolization of dopamine under conditions of DAT1 inhibition or sparse DAT1 expression. Our results do not exclude a potential extracellular orientation of other COMT isoforms that have not been characterized on protein level, but might indeed exist, based both on Western blot analyses (Tunbridge et al., 2006b) and on mRNA expression studies of novel putative COMT splice variants (Tunbridge et al., 2007). It should be noted, though, that COMT isoforms resulting from these mRNAs should be recognized by the COMT antibodies used here and thus be detected by our immunocytochemical investigations, assuming they are constitutively expressed. And indeed we could replicate the previously observed larger COMT isoform at $39 \mathrm{kDa}$, which was observed in both neurons and glial cells, although more reliably in neurons. As pointed out by Tunbridge et al. (2006b), this band most likely reflects the authentic COMT enzyme, as it was absent in COMT knockout mice. This interpretation is not in disagreement with the absence of a corresponding band in the Western blot of overexpressed COMT (Figures 4C,D), as only the open reading frame of the known MB-COMT was cloned. However, while we were able to confirm the presence of this novel COMT isoform, we still detected no COMT immunoreactivity during live staining of unpermeabilized cortical neurons and glial cells. Therefore, any potentially extracellularly active COMT isoforms would have to be expressed at very low levels or orientated to the extracellular space in a transient, possibly activity-dependent,manner.

An alternative explanation for the apparent discrepancy between intracellular orientation of MB-COMT and the well-documented role of COMT for DAT1-independent 
A

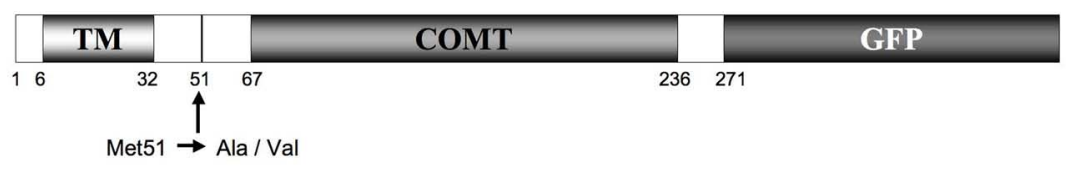

B
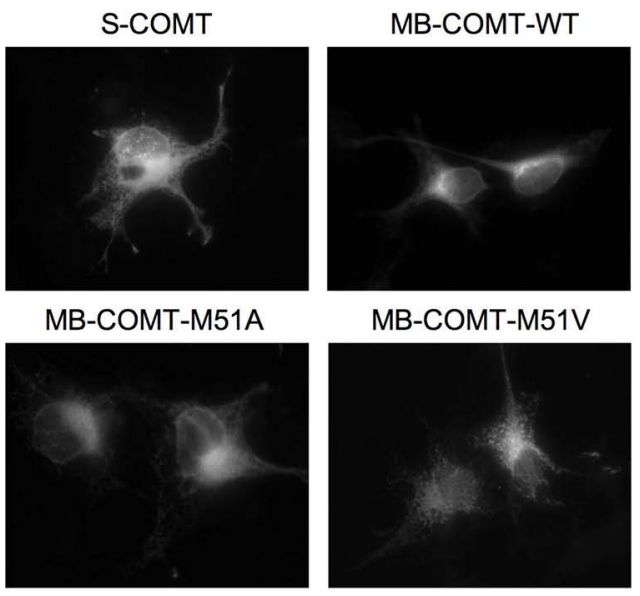

MB-COMT-M51V

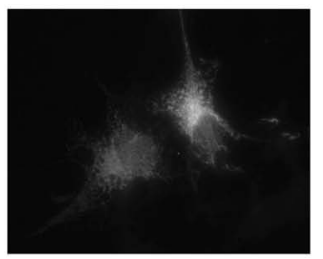

C

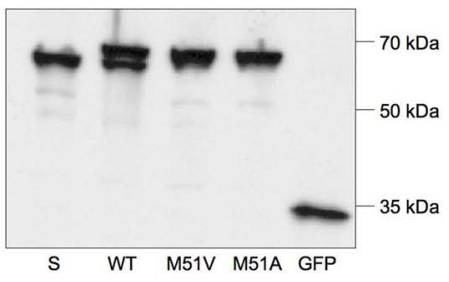

D
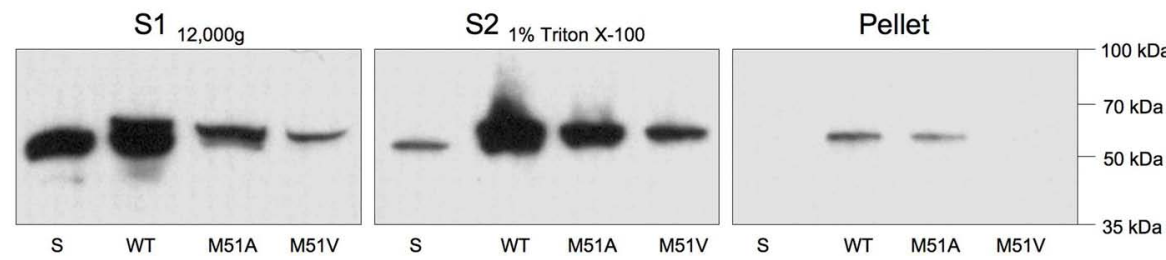

FIGURE 4 | Generation of mutated COMT-GFP fusion constructs. (A) Schematic illustration of the COMT-GFP fusion protein. The mutation site of the S-COMT start codon at Met51 is indicated. TM, putative transmembrane domain; COMT, catalytic region, present in both S- and MB-COMT; GFP, green fluorescent protein fused to the $\mathrm{C}$ terminus. (B) Autofluorescence of the
COMT-GFP fusion constructs in COS7 cells. (C) Western blot of COS7 cell homogenates, using a murine $\alpha$ GFP antibody. (D) Western blot of fractionated COS7 cell homogenates using the same antibody. S, soluble COMT;WT, wild-type MB-COMT; M51A, methionine-alanine substitution in MB-COMT; M51V, methionine-valine substitution in MB-COMT. dopamine inactivation would be the presence of additional uptake mechanisms. COMT is expressed in high concentration in the postsynaptic region of dendritic spines, suggesting the presence of a postsynaptic transporter molecule (Karhunen et al., 1995a) that might be responsible for dopamine reuptake, which could then be followed by COMT-dependent metabolization. In the PFC, dopamine can in principle be taken up by the norepinephrine transporter (NET), particularly when DAT1 is inhibited (Tanda et al., 1997; Mazei et al., 2002). However, the quantitative role of NET in dopamine uptake is under debate (Wayment et al., 2001). Because NET is, similar to DAT1, primarily expressed presynaptically (Bönisch and Brüss, 2006), NET-dependent dopamine uptake is unlikely to explain the high proportion of COMT-dependent dopamine metabolites under conditions of DAT1 inhibition.

A potential candidate transporter protein responsible for postsynaptic dopamine uptake might be the recently described plasma membrane monoamine transporter (PMAT; NCBI accession \# NM_153247), a low-affinity, high-capacity monoamine transporter with structural similarity to the nucleoside transporter family. PMAT has recently been shown to be highly expressed in both human and rodent forebrain cortex (Engel et al., 2004; Dahlin et al., 2007), including brain regions with sparse DAT1 expression. As there is to date no direct functional evidence that PMAT mediates DAT1-independent dopamine uptake in brain regions such as the PFC, future research needs to determine the functionality of PMAT in the human brain and a potential role in the pathogenesis of neurological and psychiatric disorders.

An extraneuronal monoamine transporter (EMT) has also been described. While EMT might in principle be able to transport dopamine into glial cells, where it could be metabolized via the COMT pathway, EMT expression has thus far only been detected in the area postrema of the brainstem (Haag et al., 2004), making a quantitative contribution of EMT to DAT1-independent dopamine inactivation in the PFC unlikely.

It has previously been suggested that in the PFC slower dopamine inactivation by COMT leads to a more extensive extracellular diffusion of dopamine as compared to the striatum and thus to local spreading of dopamine action (Bilder et al., 2004; Goto and Grace, 2007). While this model has previously been based on assumed 


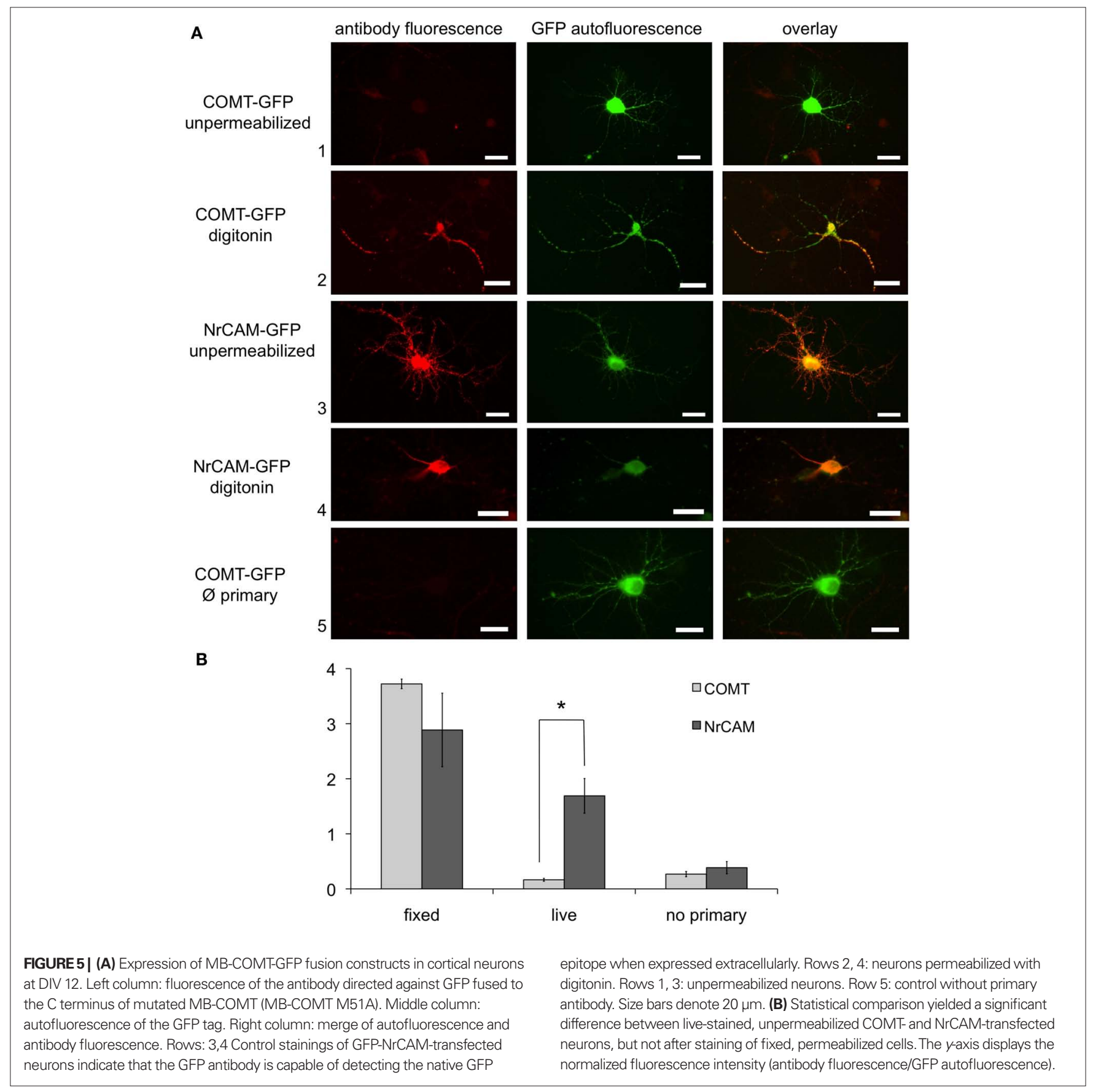

extracellular action of COMT (Bilder et al., 2004), our current findings are not incompatible with this view. Regulation of synaptic vs. extrasynaptic localization of PMAT might, for example, provide a cellular mechanism that could modulate the extent of dopamine diffusion in brain regions with low DAT1 expression.

In summary, our results show that the catalytic domain of MB-COMT is orientated toward the intracellular space, warranting further investigation of DAT1-independent dopamine uptake mechanisms in postsynaptic neurons and glial cells and the possible existence of other COMT isoforms that might act extracellularly.

\section{ACKNOWLEDGMENTS}

We thank Olivier Thoumine, Julien Falk, and Martin Heine for providing the NrCAM-GFP construct and Sabine Opitz, Monika Marunde, Heidi Wickborn, and Maria Michelmann for expert technical assistance. The authors gratefully acknowledge financial support by the Deutsche Forschungsgemeinschaft (DFG SFB 779, TP A8 \& B9 to Björn H. Schott, Constanze I. Seidenbecher, and Eckart D. Gundelfinger, and DFG Gu230/5-2, 3 to Constanze I. Seidenbecher, Renato Frischknecht, and Eckart D. Gundelfinger), and the Helmholtz Gemeinschaft (VH-VI-125). 


\section{REFERENCES}

Banker, G., and Goslin, K. (1988). Developments in neuronal cell culture. Nature 336, 185-186.

Bilder, R. M., Volavka, J., Lachman, H. M., and Grace A. A. (2004). The catecholO-methyltransferase polymorphism: relations to the tonic-phasic dopamine hypothesis and neuropsychiatric phenotypes. Neuropsychopharmacology 29, 1943-1961.

Bonisch, H., and Bruss, M. (2006). The norepinephrine transporter in physiology and disease. Handb. Exp. Pharmacol. 175, 485-524.

Chen, J., Lipska, B. K., Halim, N., Ma, Q. D., Matsumoto, M., Melhem, S., Kolachana, B. S., Hyde, T. M., Herman, M. M.,Apud, J., Egan, M. F., Kleinman, J. E., and Weinberger D. R. (2004). Functional analysis of genetic variation in catechol-O-methyltransferase (COMT): effects on mRNA, protein, and enzyme activity in postmortem human brain. Am. J. Hum. Genet. 75, 807-821

Dahlin, A., Xia, L., Kong, W., Hevner, R., and Wang J. (2007). Expression and immunolocalization of the plasma membrane monoamine transporter in the brain. Neuroscience 146, 1193-1211.

Diaz-Asper, C. M., Goldberg, T. E., Kolachana, B. S., Straub, R. E., Egan, M. F., and Weinberger D. R. (2008). Genetic variation in catechol-Omethyltransferase: effects on working memory in schizophrenic patients, their siblings, and healthy controls. Biol. Psychiatry 63, 72-79.

Egan, M. F., Goldberg, T. E., Kolachana, B. S., Callicott, J. H., Mazzanti, C. M., Straub, R. E., Goldman, D., and Weinberger, D. R. (2001). Effect of COMT Val108/158 Met genotype on frontal lobe function and risk for schizophrenia. Proc. Natl. Acad. Sci. U.S.A. 98, 6917-6922.

Elofsson, A., and von Heijne, G. (2007). Membrane protein structure: prediction versus reality. Anпu. Rev. Biochem. $76,125-140$.

Engel, K., Zhou, M., and Wang, J. (2004). Identification and characterization of a novel monoamine transporter in the human brain. J. Biol. Chem. 279, 50042-50049.

Frischknecht, R., Heine, M., Perrais, D., Seidenbecher, C. I., Choquet, D., and Gundelfinger, E. D. (2009). Brain extracellular matrix affects AMPA receptor lateral mobility and shortterm synaptic plasticity. Nat. Neurosci. $12,897-904$
Goto, Y., and Grace, A. A. (2007). The dopamine system and the pathophysiology of schizophrenia: a basic science perspective. Int. Rev. Neurobiol. 78C, 41-68.

Haag, C., Berkels, R., Grundemann, D., Lazar, A., Taubert, D., and Schomig, E. (2004). The localisation of the extraneuronalmonoamine transporter (EMT) in rat brain. J. Neurochem. 88 , 291-297.

Huotari, M., Gainetdinov, R., and Männistö, P. T. (1999). Microdialysis studies on the action of tolcapone on pharmacologically-elevated extracellular dopamine levels in conscious rats. Pharmacol. Toxicol. 85, 233-238.

John, N., Krügel, H., Frischknecht, R. Smalla, K. H., Schultz, C., Kreutz, M.R., Gundelfinger, E. D., and Seidenbecher, C. I. (2006). Brevican-containing perineuronal nets of extracellular matrix in dissociated hippocampal primary cultures. Mol. Cell. Neurosci. 31, 774-784.

Karhunen, T., Tilgmann, C., Ulmanen, I., and Panula, P. (1995a). Neuronal and non-neuronal catechol-O-methyltransferase in primary cultures of rat brain cells. Int. J. Dev. Neurosci. $13,825-834$.

Karhunen, T., Tilgmann, C., Ulmanen, I., and Panula, P. (1995b). CatecholO-methyltransferase (COMT) in rat brain: immunoelectron microscopic study with an antiserum against rat recombinant COMT protein. Neurosi. Lett. 187, 57-60.

Karoum, F., Chrapusta, S. J., and Egan, M. F. (1994). 3-Methoxytyramine is the major metabolite of released dopamine in the rat frontal cortex: reassessment of the effects of antipsychotics on the dynamics of dopamine release and metabolism in the frontal cortex, nucleus accumbens, and striatum by a simple two pool model. $J$. Neurochem. 63, 972-979.

Matsumoto, M., Weickert, C. S., Akil, M., Lipska, B. K., Hyde, T. M., Herman, M. M., Kleinman, J. E., and Weinberger, D. R. (2003). Catechol O-methyltransferase mRNA expression in human and rat brain: evidence for a role in cortical neuronal function. Neuroscience 116, 127-137.

Mazei, M. S., Pluto, C. P., Kirkbride, B., and Pehek, E. A. (2002). Effects of catecholamine uptake blockers in the caudate-putamen and subregions of the medial prefrontal cortex of the rat. Brain Res. 936, 58-67.

Reenilä, I., and Männistö, P. T. (2001). Catecholamine metabolism in the brain by membrane-bound and soluble catechol-o-methyltransferase (COMT) estimated by enzyme kinetic values. Med. Hypotheses 57, 628-632.

Rivett, A. J., Francis, A., and Roth, J. A (1983a). Localization of membranebound catechol-O-methyltransferase. J. Neurochem. 40, 1494-1496.

Rivett, A. J., Francis, A., and Roth, J. A. (1983b). Distinct cellular localization of membrane-bound and soluble forms of catechol-O-methyltransferase in brain. J. Neurochem. 40 , 215-219.

Schott, B. H., Seidenbecher, C. I., Fenker D. B., Lauer, C. J., Bunzeck, N. Bernstein, H. G., Tischmeyer, W. Gundelfinger, E. D., Heinze, H. J., and Düzel, E. (2006). The dopaminergic midbrain participates in human episodic memory formation: evidence from genetic imaging. J. Neurosci. 26 , 1407-1417.

Seidenbecher, C. I., Smalla, K. H., Fischer, N., Gundelfinger, E. D., and Kreutz, M R. (2002). Brevican isoforms associate with neural membranes. J. Neurochem. $83,738-746$.

Sesack, S. R., Hawrylak, V. A., Matus, C., Guido, M. A., and Levey, A. I. (1998) Dopamine axon varicosities in the prelimbic division of the rat prefrontal cortex exhibit sparse immunoreactivity for the dopamine transporter. $J$. Neurosci. 18, 2697-2708.

Tan, H. Y., Chen, Q., Goldberg, T. E. Mattay, V. S., Meyer-Lindenberg, A. Weinberger, D. R., and Callicott, J. H. (2007). Catechol-O-methyltransferase Val158Met modulation of prefrontalparietal-striatal brain systems during arithmetic and temporal transformations in working memory. J. Neurosci. 27, 13393-13401.

Tanda, G., Pontieri, F. E., Frau, R., and Di Chiara, G. (1997). Contribution of blockade of the noradrenaline carrier to the increase of extracellular dopamine in the rat prefrontal cortex by amphetamine and cocaine. Eur. $J$. Neurosci. 9, 2077-2085.

Thoumine, O., Saint-Michel, E., Dequidt C., Falk, J., Rudge, R., Galli, T., FaivreSarrailh, C., and Choquet, D. (2005). Weak effect of membrane diffusion on the rate of receptor accumulation at adhesive contacts. Biophys. J. 89, L40-L42.

Tunbridge, E. M., Harrison, P. J., and Weinberger, D. R. (2006a). Catecholo-methyltransferase, cognition, and psychosis: Val158Met and beyond. Biol. Psychiatry 60, 141-151.
Tunbridge, E. M., Weinberger, D. R., and Harrison P. J. (2006b). A novel protein isoform of catechol $\mathrm{O}$-methyltransferase (COMT): brain expression analysis in schizophrenia and bipolar disorder and effect of Val158Met genotype. Mol. Psychiatry 11, 116-117.

Tunbridge, E.M.,Lane, T.A., and Harrison, P.J. (2007). Expression of multiple catechol-o-methyltransferase (COMT) mRNA variants in human brain. Am J. Med. Genet. B Neuropsychiatr. Genet. 144, 834-839.

Ulmanen, I., Peranen, J., Tenhunen, J., Tilgmann, C., Karhunen, T., Panula P., Bernasconi, L., Aubry, J. P., and Lundstrom, K. (1997). Expression and intracellular localization of catechol O-methyltransferase in transfected mammalian cells. Eur. J. Biochem. $243,452-459$.

Wayment, H. K., Schenk, J. O., and Sorg, B. A. (2001). Characterization of extracellular dopamine clearance in the medial prefrontal cortex: role of monoamine uptake and monoamine oxidase inhibition. J. Neurosci. 21, 35-44.

Williams, H. J., Owen, M. J., and O'Donovan, M. C. (2007). Is COMT a susceptibility gene for schizophrenia? Schizophr. Bull. 33, 635-641.

Conflict of Interest Statement: The authors declare that the research was conducted in the absence of any commercial or financial relationships that could be construed as a potential conflict of interest.

Received: 02 July 2010; accepted: 29 September 2010; published: 28 October 2010.

Citation: Schott BH, Frischknecht $R$, Debska-Vielhaber G, John N, Behnisch G, Düzel E, Gundelfinger ED and Seidenbecher CI (2010) Membrane-bound catecholO-methyl transferase in cortical neurons and glial cells is intracellularly oriented. Front. Psychiatry 1:142. doi: 10.3389/ fpsyt.2010.00142

This article was submitted to Frontiers in Molecular Psychiatry, a specialty of Frontiers in Psychiatry.

Copy right ( 2010 Schott, Frischknecht, Debska-Vielhaber, John, Behnisch, Düzeh, Gundelfinger and Seidenbecher. This is an open-access article subject to an exclusive license agreement between the authors and the Frontiers Research Foundation, which permits unrestricted use, distribution, and reproduction in any medium, provided the original authors and source are credited. 